\title{
Consumo De Drogas Y Estrés En Estudiantes De Medicina De La Ciudad De Querétaro, México
}

\author{
Diana Laura Sánchez Camacho, \\ Estudiante pasante de la Licenciatura en Enfermería, \\ Universidad Autónoma de Querétaro, Querétaro, México \\ María Magdalena Gachuzo Elías, \\ Estudiante pasante de la Licenciatura en Enfermería, \\ Universidad Autónoma de Querétaro, Querétaro, México \\ Ruth Magdalena Gallegos Torres, \\ Doctora en Ciencias de la Salud \\ Universidad Autónoma de Querétaro, Querétaro, México
}

Doi:10.19044/esj.2021.v17n21p366

Submitted: 17 March 2021

Accepted: 29 May 2021

Published: 30 June 2021
Copyright 2021 Author(s)

Under Creative Commons BY-NC-ND 4.0 OPEN ACCESS

Cite As:

Sánchez Camacho D.L., Gachuzo Elías M.M. \& Gallegos Torres R.M. (2021). Consumo De Drogas Y Estrés En Estudiantes De Medicina De La Ciudad De Querétaro, México. European Scientific Journal, ESJ, 17(21), 366.

https://doi.org/10.19044/esj.2021.v17n21p366

\section{Resumen}

Introducción. La adicción a drogas lícitas e ilícitas constituye un problema de salud pública que se presenta tanto a nivel nacional como internacional. Las escuelas de medicina no están ajenas a este fenómeno, dado que los alumnos están sometidos a altas demandas académicas que se asocian, en algunos casos, a estrés, ansiedad y trastornos del ánimo. Objetivo. Identificar la prevalencia y tipo de drogas consumidas en estudiantes universitarios de la Licenciatura en Medicina, así como las características del estrés académico presente. Metodología. Estudio con enfoque cuantitativo, con un diseño descriptivo y trasversal, realizado con estudiantes de quinto hasta décimo semestre. Se aplicó la "Encuesta Nacional sobre Prevención y Uso de Drogas" conformada por 7 secciones, de 65 preguntas y el inventario SISCO de estrés académico. Los datos fueron procesados mediante estadística descriptiva. El proyecto se evaluó y registró ante un comité de investigación. Se cuidaron los aspectos éticos. Resultados. Se aplicaron 177 cuestionarios, 
58.8\% fueron mujeres. En cuanto al consumo de sustancias, $75.1 \%$ ha fumado y $99.4 \%$ ha consumido alcohol. El $66.1 \%$ ha consumido marihuana y $13.6 \%$ cocaína. Del Inventario SISCO, 97.2\% presentó momentos de preocupación o nerviosismo durante el semestre, teniendo distintos tipos de reacciones como: fatiga, somnolencia o necesidad de dormir 34\%, depresión y tristeza (39\% algunas veces y 19.2\% casi siempre). Conclusiones. La información obtenida plantea la posibilidad de la creación de un programa para reducir el estrés y sus manifestaciones con el fin de disminuir el consumo de drogas y preservar la salud física y mental de los alumnos.

Palabras-clave: Drogas, Estrés Académico, Estudiantes

\title{
Drug Consumption And Stress In Medicine Students From Queretaro City, Mexico
}

Diana Laura Sánchez Camacho, Estudiante pasante de la Licenciatura en Enfermería, Universidad Autónoma de Querétaro, Querétaro, México

María Magdalena Gachuzo Elías, Estudiante pasante de la Licenciatura en Enfermería, Universidad Autónoma de Querétaro, Querétaro, México Ruth Magdalena Gallegos Torres, Doctora en Ciencias de la Salud Universidad Autónoma de Querétaro, Querétaro, México

\begin{abstract}
Introduction. Addiction to legal and illegal drugs represent a public health problem that take place nationally and internationally. Medical schools are not immune to this phenomenon, since students are expose to high academic demands that are associated, in some cases, with stress, anxiety and mood disorders. Objective. Identify the prevalence and type of drugs consumed by medical students, as well as the characteristics of the academic stress. Methodology. Quantitative, descriptive, cross-sectional study. Carried out with students from fifth to tenth semester. The "National Survey on Prevention and Use of Drugs" was applied, made up of seven sections, with 65 questions and the SISCO inventory of academic stress. The information was processed using descriptive statistics. Results. 177 questionnaires were applied, 58.8\% were women. Regarding substance use, $75.1 \%$ have smoked and $99.4 \%$ have consumed alcohol. $66.1 \%$ have consumed marijuana and $13.6 \%$ cocaine. From the SISCO Inventory, 97.2\% presented moments of panic or nervousness during the semester, having different types of reactions such as: fatigue, drowsiness or need to sleep 34\%, depression and sadness
\end{abstract}


(39\% sometimes and 19.2\% almost always). Conclusions. The information obtained allows us to suggest the creation of a program to reduce stress and its manifestations in order to lessen drug use and preserve the physical and mental health of students.

Keywords: Drugs, Academic Stress,Students

\section{Introducción}

Durante los últimos años, el estado de salud a nivel mundial ha ido deteriorándose, lo que ha generado múltiples emergencias causadas por conflictos, catástrofes naturales o brotes de enfermedades. Muchas de estas crisis podrían evitarse por completo y, a menudo, son producto de la actividad humana, ya que son más las muertes por enfermedades que se podrían prevenir y tratar o por enfermedades crónicas que las causadas por la violencia en sus diversas manifestaciones (Organización Mundial de la Salud, 2018).

$\mathrm{Al}$ respecto, en México se han observado cambios trascendentes en el comportamiento epidemiológico de las enfermedades (Soto, et al., 2016). Se han modificado las formas de vida y surgido riesgos de exposición al sedentarismo, al estrés, al consumo de tabaco y de drogas, así como a la violencia, patrones alimentarios compuestos por alimentos de alta densidad energética, el sobrepeso y la obesidad, el colesterol elevado y la hipertensión arterial, factores responsables de gran parte de la carga global de la enfermedad, ya que han dado como resultado problemas como la obesidad y otras enfermedades crónico degenerativas a edades cada vez más tempranas (Ezzati \& Riboli, 2013).

La adicción a drogas lícitas e ilícitas constituye un problema de salud pública que tiene muchos años y continua vigente, que se presenta tanto a nivel nacional como internacional. Este fenómeno afecta de manera específica a la población de niños, adolescentes y jóvenes, quienes son particularmente vulnerables; de la misma manera, no hay distinción de género (Instituto Nacional de Salud Pública, 2013; Villatoro, et al., 2011).

Las drogas ilícitas o ilegales, son reconocidas como las sustancias que tienen un objeto de prohibición legal dados los efectos dañinos que generan en el cuerpo. Por otro lado, las drogas lícitas o legales, están permitidas por la ley y su máxima prohibición para su uso podría ser la edad de consumo (Comisión Nacional para el Desarrollo y Vida sin Drogas, 2009).

Los efectos de las sustancias lícitas e ilícitas son variados en el organismo. Además de que depende del nivel de sensibilidad de cada personal, influye el efecto reconocido de estas en el organismo, es decir, depresor, estimulante o alucinógeno (Comisión Nacional para el Desarrollo y Vida sin Drogas, 2009). 
El estudio del consumo de drogas desde el ámbito universitario constituye una oportunidad para desarrollar estrategias de prevención acorde a las características de los estudiantes que la conforman, a su filosofía, al ámbito social, político y económico que se cuenta (Sánchez, Andueza, Santana, Hoil \& CuFarfán, 2017).

Las escuelas de medicina no están ajenas a este fenómeno. Existe numerosa bibliografía internacional sobre el consumo de antidepresivos, psicotrópicos y marihuana (Akvard, Demiral, Ergor, Ergor, Bilici \& Ozer, 2003; McAuliffe, Rohman, Breer, Wyshak, Santangelo \& Magnuson, 1991). Con un enfoque nacional, en el 2016, en la Universidad Veracruzana se encontró como parte de un estudio, que en estudiantes de medicina existe un consumo elevado de drogas ilícitas (Arellanez, De San Jorge, Salas \& Beverido, 2016).

Iniciar una carrera universitaria puede ser motivante, sin embargo, en algunos casos resulta ser una experiencia estresante, difícil de manejar, y se agudiza más si se estudia una profesión relacionada con las ciencias de la salud, convirtiéndose los estudios en una fuente generadora de estrés; éste puede presentarse cuando la persona experimenta tensión en su proceso de aprendizaje, desde los grados preescolares hasta la educación universitaria y de posgrado (Zárate, Soto, Martínez, Castro, García \& López, 2018).

La presencia del estrés en estudiantes de todos los niveles y edades es una realidad que acontece en las instituciones académicas. Las Ciencias Médicas han sido reportadas como una de las ramas donde los estudiantes manifiestan mayores niveles de estrés. Según Carmel y Bernstein, la escuela de medicina es un ambiente muy estresante (Román, Ortiz \& Hernández, 2008).

El estrés originado en el contexto educativo suele denominarse estrés académico, estrés escolar, estrés de examen, entre otros sinónimos (Berrío \& Mazo, 2011). Para Barraza (2005) el estrés académico es aquel que padecen los alumnos de educación media superior y superior y que tiene como fuente exclusiva a estresores relacionados con las actividades a desarrollar en el ámbito escolar.

La gran mayoría de estudiantes universitarios experimentan un grado elevado de estrés académico, pues tienen la responsabilidad de cumplir las obligaciones académicas; experimentan en ocasiones sobrecarga de tareas y trabajos, además de la evaluación de los profesores, de sus padres, y de ellos mismos sobre su desempeño, lo que genera ansiedad y puede redundar de modo negativo tanto en el rendimiento en los diferentes compromisos, como en la salud física y mental de los estudiantes (Berrío \& Mazo, 2011).

De manera extraoficial, entre estudiantes, se conoce que hay manejo, venta y consumo de sustancias en la universidad. Al respecto, se señala, que por el nivel de estrés, los tiempos invertidos para estudiar, entre otros, los 
alumnos de medicína no están excentos del consumo, por lo que el estudio es trascendente y relevante, ya que muestra cuan vigente es este fenómeno.

Al ser una problemática frecuente, es importante investigar el fenómeno, de manera tal que el objetivo del estudio fue Identificar la prevalencia y tipo de drogas consumidas en estudiantes universitarios de la Licenciatura en Medicina de la Facultad de Medicina de la Universidad Autónoma de Querétaro, estableciendo las características del estrés académico presente, partiendo de señalar primeramente los datos sociodemográficos, detalles del consumo de tabaco y alcohol, luego de drogas ilegales y refererir los datos de estrés académico.

\section{Metodología}

Se trató de un estudio de corte cuantitativo, transversal y descriptivo (Hernández \& Mendoza, 2018). Se realizó con estudiantes de la Facultad de Medicina de la Universidad Autónoma de Querétaro. Dicha facultad cuenta con la Licenciatura en Medicina y Licenciatura en Odontología, más una carrera en Técnico Superior en Prótesis Dental. Se trabajó únicamente con los estudiantes en la Licenciatura en Medicina que ya tenían un contacto previo con las unidades hospitalarias, por lo que se tomaron a los grupos a partir del 5to. a 10mo. semestre, siendo un un total de 360 alumnos aproximadamente. Para el cálculo del tamaño de la muestra se utilizó una fórmula para poblaciones finitas, con un margen de error esperado del 6\%, un nivel de confianza del 95\%, lo que arroja un tamaño de muestra aproximado de 186 individuos, el cual fue planteado en cerrar a 200.

El tipo de muestreo fue por conveniencia, dado que las autoridades de la institución determinarían a los grupos con los que se trabajaría. Los participantes fueron elegidos con base en los criterios de inclusión y con previa autorización por medio del consentimiento informado.

El estudio se aprobó ante un Comité de Investigación y un Comité de Bioética, quienes evaluaron y aprobaron los principios éticos del estudio, con base en la Ley General de Salud en materia de Investigación para la Salud, el Código de Nuremberg y la Declaración de Helsinki.

Con respecto al cuestionario para medir el consumo de drogas, se utilizó la Encuesta Nacional sobre Prevención y Uso de Drogas, la cual es la primera encuesta nacional oficial sobre la epidemiología de las drogas entre la población urbana de Perú. Ha sido coordinada por la Unidad de Prevención y Rehabilitación de contradrogas y ejecutada por el Instituto Nacional de Estadística e Informática (INEI) dentro del Convenio Marco de Cooperación Técnica Interinstitucional entre CONTRADROGAS y el INEI, suscrito el 25 de junio de 1998.

Para lograr recabar los datos de interés y de acuerdo a los objetivos de la investigación, se realizó una adaptación a la encuesta ya mencionada por 
parte de las investigadoras y directora de tesis; dicha adaptación consistió en eliminar preguntas relacionadas con aspectos de características de vivienda de los participantes o de datos sociales no relacionados con el aspecto del consumo de drogas. Debido a los cambios que se hicieron en el instrumento, éste se sometió a un proceso de validación tomando en cuenta las opiniones y sugerencias de personas con alta experiencia en el tema, que en su caso fueron 5 personas con grado de especialidad o maestría, todo esto a través de la técnica Delphi. El proceso no arrojó observaciones de relevancia que implicaran ajustes mayores al formato del cuestionario.

En este sentido, el documento quedó conformado de 7 secciones con un total de 65 preguntas, las cuales se desglosan a continuación:

\begin{tabular}{|c|c|c|}
\hline Secciones & Nombre & $\mathbf{N}^{\circ}$ de peguntas \\
\hline 1 & Datos sociodemográficos & 5 \\
\hline II & Relaciones familiares. & 6 \\
\hline III & Relaciones personales & 10 \\
\hline IV & Activdades & 4 \\
\hline V & Salud & 19 \\
\hline VI & Consumo de tabaco y alcohol & 16 \\
\hline VII & Consumo de otras drogas & $\mathbf{6 5}$ \\
\hline \multicolumn{2}{|c|}{} \\
\hline
\end{tabular}

Así mismo, se aplicó el inventario SISCO de Estrés académico (Barraza, 2007) el cual tiene como objetivo identificar las características del estrés presentes en los estudiantes durante su formación; es autoadministrado y está constituido por 31 ítems. Inicialmente cuenta con un ítem de filtro, el cual es dicotómico y el resto se encuentra en un escalamiento tipo Likert. Dicho inventario se encuentra validado, tiene una confiabilidad por mitades de $.87 \mathrm{y}$ en alfa de Cronbach de .90 .

Para el procesamiento de los datos se utilizó la estadística descriptiva, dentro de esta se obtuvieron valores en porcentajes y frecuencias; de igual forma se utilizaron medidas de tendencia central que incluyen la moda, mediana y media, también se incluyeron las medidas de dispersión, rango, desviación estándar y la varianza, a través del paquete SPSS (Statistical Package for the Social Sciences) versión 20 en español, bajo licencia de la Facultad de Enfermería de la Universidad Autónoma de Querétaro.

\section{Resultados}

La muestra final estuvo conformada por 177 estudiantes. 58.8\% fueron mujeres y $41.2 \%$ hombres. Sobresalieron las edades de 21 a 23 años de quinto hasta décimo semestre. 26 de ellos trabajan y el resto solo se dedican a las tareas académicas.

Con respecto al consumo de sustancias, referido específicamente a las sustancias legales que son el tabaco y alcohol, han fumado alguna vez 75.1\% 
y en los últimos 30 días previos al estudio, el 32.2\% de los participantes. Las edades de inicio del consumo de la mayoría de los estudiantes, fue de los 14 los 18 años. El aspecto impulsor para el consumo fue, en el 76\% de los casos la curiosidad. Con respecto a cada cuándo fuman, 30\% lo hacen diario y $47 \%$ en las fiestas.

Han tomado alcohol alguna vez el $99.4 \%$; de estos, $60 \%$ lo hizo en los últimos 30 días previos a la investigación, habiendo iniciado el consumo entre los 15 y 18 años, donde la curiosidad influyó en el 55.9\%. En relación a cada cuándo toman, el 10.7\% señaló que todos los días y 13.6\% en las fiestas.

De las drogas ilegales que se abordaron, se contemplaron la marihuana, cocaína, éxtasis, anfetaminas, entre otras. Relacionado con esto, $28 \%$ señala que las probarían para ver qué se siente. $73.4 \%$ ha interactuado con la marihuana, $16.9 \%$ con cocaína, $14.1 \%$ con tranquilizantes, $10.2 \%$ con LSD y $1.1 \%$ con cristal.

De los resultados anteriores, la primera vez que consumieron tenían un rango de edad entre 16 a 22 años y se realizó estando entre un grupo de amigos; actualmente un $60.4 \%$ continua con el consumo de alguna de las sustancias ya mencionadas entre hace más de un año y/o menos de un año, y el motivo que destacó fue: por pasarla "bien", al igual que destaca que el principal motivo de continuar con el consumo es por "pasarla bien” y "porque me gusta".

Ahora bien, al sondear la presencia de estrés con el Inventario SISCO, sobre todo el vínculo con el aspecto académico, los datos muestran los siguiente: un 97.2\% confirma haber tenido momentos de preocupación y nerviosismo durante el semestre.

En una escala de 1 a 5 para valorar la intensidad del estrés sentido, donde 1 es poco estrés y 5 máximo estrés, 18.1\% señalaron 5 y $40.1 \%$ un valor de 4. La sobrecarga de tareas y los trabajos escolares les estresa, casi siempre, al 37\%; las evaluaciones de los profesores, siempre, al 36.2\%.

Como manifestaciones del estrés, los alumnos refirieron que han presentado fatiga crónica: siempre, el 24\% y casi siempre 30\%. Rascarse, morderse las uñas, frotarse, el $25.4 \%$ casi siempre. Somnolencia o necesidad de dormir, 34\% siempre. Sentimientos de depresión y tristeza (decaído), 39\% algunas veces; $19.2 \%$ casi siempre. Ansiedad, angustia o desesperación, 29.9\% algunas veces, 33.9\% casi siempre. Desgano para realizar labores escolares $31.6 \%$ algunas veces. Aumento o reducción del consumo de alimentos 35\% casi siempre.

En lo que respecta a las reacciones tanto físicas, psicológicas y comportamentales que sufren los estudiantes cuando están preocupados o nerviosos los resultados se muestran en la gráfica $1,2$. 
Gráfica 1. Reacciones físicas ante el estrés, referidas por los alumnos

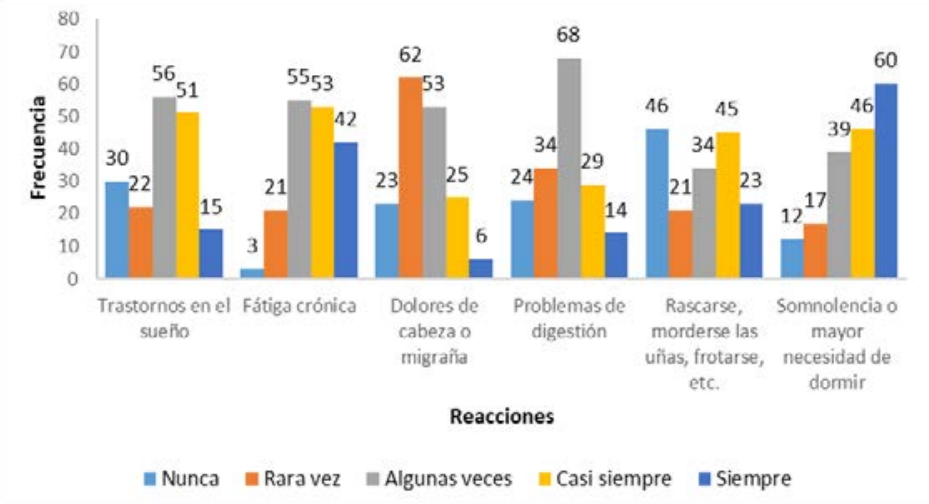

\section{Fuente: Cuestionario}

Gráfica 2. Reacciones psicológicas ante el estrés, referidas por los alumnos

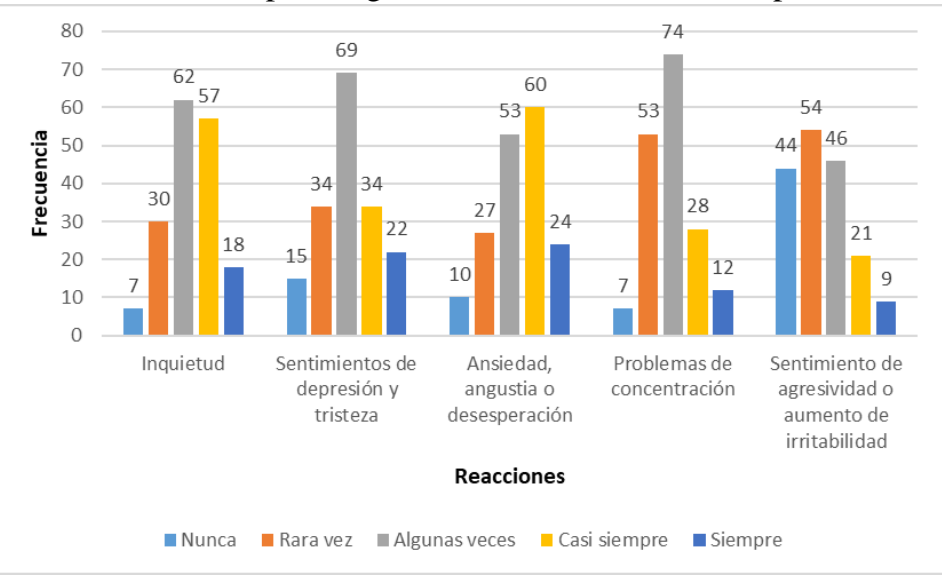

Fuente: Cuestionario

Gráfica 3. Reacciones comportamentales ante el estrés, referidas por los estudiantes

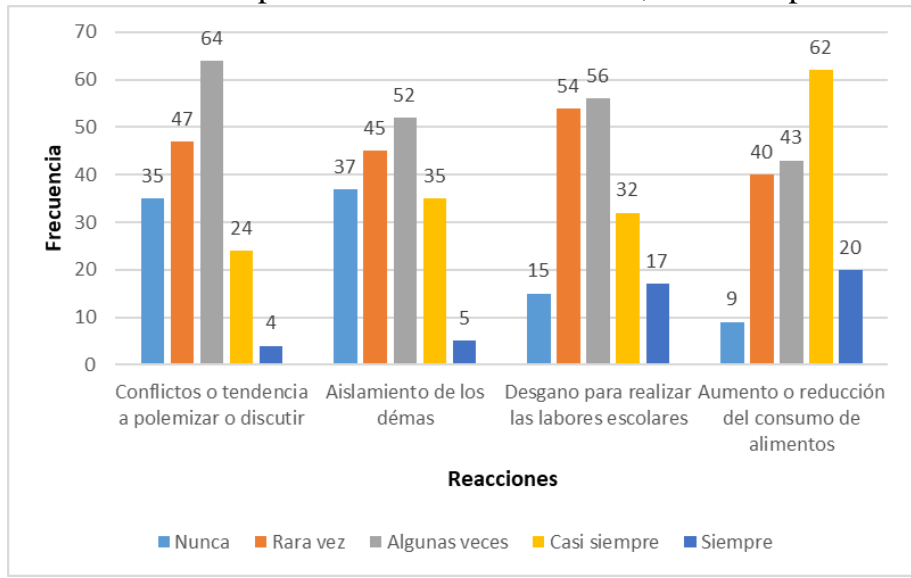

Fuente: Cuestionario 
Se añadió un último apartado para identificar las estrategias empleadas para enfrentar las situaciones que generan preocupación y nerviosismo en los alumnos, destaco: elaboración de un plan y ejecución de tareas, $44.6 \%$ casi siempre. Elogios a sí mismo, 33.3\%, algunas veces. Verbalización de la situación, 46.9\% casi siempre (gráfica 4).

Gráfica 4. Estrategias referidas por los estudiantes, como medio para afrontar el estrés

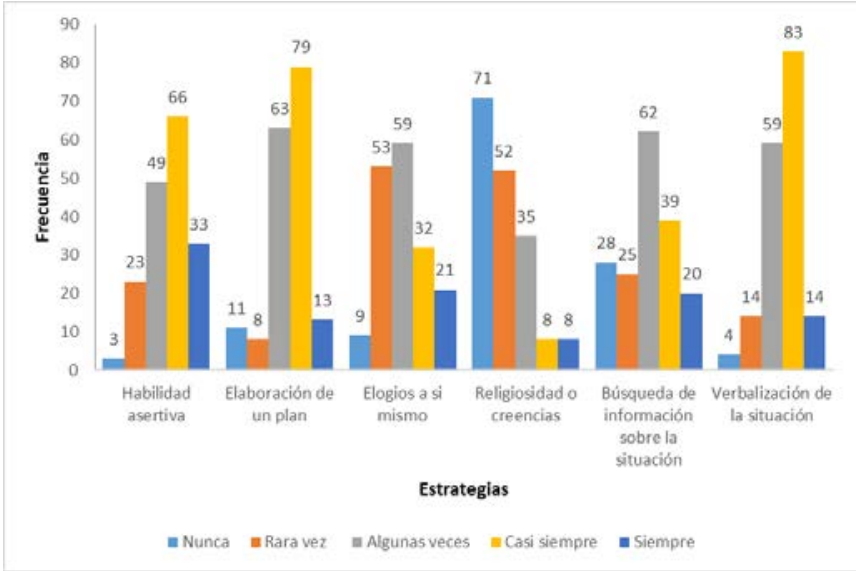

Fuente: cuestionario administrado

\section{Discusión}

Los hallazgos obtenidos muestran que un gran porcentaje de alumnos han consumido algún tipo de droga legal, tomando en cuenta el tabaco y alcohol con un consumo del $75.1 \%$ y $99.4 \%$ respctivamente, lo cual se relaciona con Arellanez, De San Jorge, Salas y Beverido en un estudio realizado en la Universidad Veracuzana en estudiantes universitarios de Ciencias de la Salud en el 2016, el cual arrojó que más de la mitad de los estudiantes han fumado alguna vez en su vida y poco más del $80 \%$ han consumido alcohol, siendo esta la sustancia de mayor prevalencia dentro de ambas investigaciones, sin embargo el consumo en la Universidad Autónoma de Querétaro se encuentra por arriba con respecto a la de Veracruz. En el trabajo de Velásquez y Mejía, (2009) en la Universidad de Antioquia se precisa que el consumo de riesgo de alcohol genera mayor probabilidad de presentar bajo rendimiento académico.

Ahora bien, dentro de las drogas ilegales se menciona en los resultados que la de mayor consumo es la marihuana con un $73.4 \%$, lo que tiene una diferencia significativa con la investigación realizada por Romero, Santander, Hitschfeld, Labbé, y Zamora, (2009) en la Universidad Católica de Chile, la cual arroja que de 569 estudiantes de medicina encuestados, el 33\% ha probado la marihuana alguna vez en su vida. Aunque el consumo fue menor, pero no menos importante de cocaína con un $16.9 \%$, tranquilizantes con un $14.1 \%$, LSD con un $10.2 \%$ y cristal con un $1.1 \%$, es un porcentaje en general 
alto comparado nuevamente con los resultados obtenidos de Arellanez, De San Jorge, Salas y Beverido en un estudio realizado en la Universidad Veracuzana, donde el consumo de drogas ilicitas durante los doce meses previos a su participación en la encuesta mostró que el $8.4 \%$ de los estudiantes habían consumido al menos una droga ilícita.

En la investigación realizada por De San Jorge, Beverido, Salas, Cruz, Roa, \& Rubiano (2017) en dos universidades latinoamericanas, se arroja que en la universidad mexicana investigada, las drogas ilegales que mostraron una tendencia a afectar de manera negativa el rendimiento académico fueron la marihuana, la cocaína y los inhalables. En el caso específico de la marihuana, los trabajos de Velázquez y Mejía, (2009) y de Font-Mayolas et al. (2006) coinciden con los hallazgos en la Universidad Veracruzana, así como en el caso de la cocaína, cuya relación también fue encontrada en el trabajo de Caso \& Hernández (2007); en el consumo de ambas drogas se detectó una relación con el bajo rendimiento académico. Por el contrario, el trabajo de Magaña (2007) no encontró asociación entre ambas variables.

En el caso del estrés académico, es evidente el problema presente en los estudiantes universitarios, con una prevalencia mayor al $90 \%$, lo cual es muy alarmante. Un estudio realizado en estudiantes de medicina de la Universidad Católica de Cuenca-Ecuador en 2017 (Santos, Jaramillo, Morocho, Senín \& Rodríguez, 2017), reportaron 95\% de estrés (151estudiantes); la investigación realizada por Obregón, Montalván, Segama, Dámaso, Panduro y Arteaga (2020) en la Universidad Nacional Hermilio Valdizán de Huánuco, Perú en (2018) registro 96\% de estrés en su estudio de 179 participantes de la Facultad de Medicina y otra investigación realizada en (2018) por García, Hernández, Och, Cocom \& García, con estudiantes de medicina de la Universidad de Quintana Roo anuncio que el 100\% de sus encuestados sufrió momentos de estrés durante su formación académica, cabe mencionar que todos estos estudios utilizaron el inventario SISCO, y además concluyen que este fenómeno actúa como factor de riesgo para la salud física y emocional del alumno.

En los resultados obtenidos en las categorías Reacciones físicas, Reacciones psicológicas y Reacciones comportamentales, que se incluyen en el inventario SISCO, su mayor porcentaje se encuentra en la opción "algunas veces" seguido de "casi siempre" y "rara vez", sin embargo hay datos que resultan interesantes. La ansiedad, angustia o desesperación fue la reacción psicológica de mayor prevalencia y los sentimientos de depresión y tristeza el de menor porcentaje. Respecto a las reacciones físicas, la somnolencia o mayor necesidad de dormir fue el síntoma más frecuente en comparación con los dolores de cabeza y migraña que fue el de menor frecuencia. Estos resultados difieren muy poco con los obtenidos en un estudio realizado en tres universidades de Medellín y el Valle de Aburrá con una muestra de 450 
estudiantes Restrepo, Sánchez \& Castañeda,(2020), en esta misma investigación se encontró una similitud con los datos obtenidos en la categoría de Reacciones comportamentales ya que en ambos estudios el aumento o reducción del consumo de alimentos fue la opción con mayor porcentaje y los conflictos o tendencias a polemizar o discutir fue la opción de menor elección. Lara, Saldaña, Fernández \& Delgadillo, (2015), mencionan que el estrés presente en los alumnos tiene relación en la percepción de calidad de vida y salud ya que aumentaba el consumo de alcohol, tabaco, menor actividad física y malestares emocionales.

La sobrecarga de tareas y trabajos así como las evaluaciones de los profesores (exámenes, ensayos, trabajos de investigación, entre otros), fueron las opciones que más inquietaban a los alumnos. Lo antes mencionado tiene similitud con los datos reportados por Chiyong \& Villacampa, (2015) donde estudiaron a los universitarios de la carrera de odontología. Berrío \& Mazo (2011) reportaron en su estudio que los alumnos tienen un grado elevado de estrés académico debido a la responsabilidad de cumplir sus obligaciones académicas, experimentando en ocasiones sobrecarga de tareas y trabajos, sumando las evaluaciones de profesores, de padres y de ellos mismos sobre su desempeño, lo que genera alto nivel de ansiedad.

Las estrategias más utilizadas por los estudiantes para afrontar el estrés fueron la habilidad asertiva (defender nuestras preferencias, ideas o sentimientos sin dañas a otros) y los elogios a sí mismo, en ese orden, estos datos difieren un poco con los obtenidos por Nieves, Satchima, González \& Jiménez (2013) en la cual sus respuestas con mayor porcentaje fueron, la habilidad asertiva $40.90 \%$ y con un $35.75 \%$ la elaboración de un plan y ejecución de sus tareas. Restrepo, Sánchez y Castañeda, (2020) mencionan que los universitarios descubren una manera de combatir el estrés apropiándose y defendiendo lo que consideran correcto y que se logra desarrollar a través de su aprendizaje y cumplimiento de diferentes actividades.

En relación al manejo de estrés se menciona la terapia cognitiva Beck, (1979), en Rodríguez, Lino y Escobar, (2017), incluye la modificación de pensamientos negativos, mediante tareas distractoras, registro diario de pensamientos disfuncionales y modificación de creencias o supuestos, esto podría dar una justificación al utilizar los elogios a sí mismo, ya que los alumnos generan confianza y seguridad lo que reflejaría mayor afrontamiento a sus dificultades.

\section{Conclusión}

Los resultados muestran el evidente consumo de drogas legales como ilegales, dentro del primer grupo el alcohol y tabaco, y del segundo grupo destaca el consumo de la marihuana, la cocaína, tranquilizantes, LSD y cristal. 
Se puede considerar alarmante pues cada uno de los participantes reportó que mínimo una vez en su vida ha tenido contacto con alguna sustancia y un considerable porcentaje ha consumido sustancias que representan un verdadero peligro para la salud, y un poco más del 60\% continúa con el consumo de alguna de las sustancias mencionadas.

Ahora bien, del Inventario SISCO es importante destacar que se vive un estrés constante dentro de la carrera de medicina, lo cual los lleva a diferentes escenarios que tienen manifestaciones en forma de reacciones tanto físicas, psicológicas y comportamentales, de los cuales caben destacar la fatiga crónica, somnolencia o mayor necesidad de dormir, inquietud, ansiedad angustia o desesperación, el aislamiento de los demás y el aumento o reducción del consumo de alimentos.

Es vital pesquisar y controlar el consumo de fármacos y drogas en estudiantes de medicina, ya que son más propensos a su consumo por requerimientos académicos, porque tienen mayor facilidad para conseguirlos y porque se sabe que, ante la sociedad, todos los estudiantes del área de la salud están llamados y vistos a ser como vehículo de fomento de actitudes de vida saludable y un ejemplo en su actuar.

La información obtenida da apertura a analizar con mayor profundidad la etiología, manifestaciones clínicas, posibles tratamientos y factores relacionados respecto al consumo de drogas así como del estrés académico, y así priorizar actividades de reducción de daño en los estudiantes que pueda ocasionar disfunción en su rendimiento académico, ya que al hacerlo generará un beneficio a la población estudiantil que se reflejara en su desempeño y reconocimiento académico, se sugiere que la institución genere programas, políticas o líneas de acciones con el objetivo de disminuir la problemática aquí mencionada y crear promoción de la salud.

\section{References:}

1. Akvardar Y, Demiral Y, Ergor G, Ergor A, Bilici M, Ozer OA. (2003). Substance use in a sample of Turkish medical students. Drug Alcohol Depend 2003; 72: 117-21.

2. Arellanez J., De San Jorge X., Salas Betzaida \& Beverido P. (2016). Consumo de alcohol, tabaco y otras drogas en estudiantes universitarios de ciencias de la salud. Revista Investigaciones Sociales, Vol. $\quad 2, \quad$ No.6 38-46. https://www.ecorfan.org/republicofnicaragua/researchjournal/investig acionessociales/journal/vol2num6/Revista_Investigaciones_Sociales_ V2_N6_4.pdf

3. Barraza, A. (2005). Características del estrés académico de los alumnos de educación media superior. PsicologíaCientífica.com. http://www.psicologiacientifica.com/ bv/psicologiapdf-19- 
caracteristicas-del-estresacademico-de-los-alumnos-de-educacionmediasuperior.pdf

4. Beck, A. (1979). Terapia Cognitiva de la Depresión. New York: Desclée de Brouwer. Bilbao.

5. Berrío García, Nathaly, \& Mazo Zea, Rodrigo. (2011). Estrés Académico. Revista de Psicología Universidad de Antioquia, 3(2), 6582. Recuperado el 17 de junio de 2019, de http://pepsic.bvsalud.org/scielo.php?script=sci_arttext\&pid=S214548922011000200006\&lng=pt\&tlng=es.

6. Berrio GarcíaN., \& Mazo ZeaR. (2012). Estrés Académico. Revista De Psicología Universidad De Antioquia, 3(2), 55-82. https://revistas.udea.edu.co/index.php/psicologia/article/view/11369

7. Caso, J. y Hernández, L. (2007). Variables que inciden en el rendimiento académico de adolescentes mexicanos. Revista Latinoamericana de Psicología, 29(3), 487-501

8. Chiyong, T. y Villacampa S. (2015) Estrés y desempeño académico en estudiantes de Odontología. Odontología Sanmarquina, 18(1), 23-27. https://revistasinvestigacion.unmsm.edu.pe/index.php/odont/article/vi ew/11336

9. Comisión Nacional para el Desarrollo y Vida Sin Drogas, DEVIDA. (2009). Estudios Cualitativos sobre el Consumo de Drogas Sintéticas en Grupos de Riesgo. Informe del estudio realizado en jóvenes de las ciudades de Lima, Cusco y Trujillo, Perú. http://www.comunidadandina.org/DS/DROSICAN/estudios\%20cuali tativos/cualit ativo\%20Per\%C3\%BA\%20ok.pdf

10. Ezzati M \& Riboli E. (2013). Behavioral and dietary risk factors from noncommunicable diseases. N Engl J Med. 2013; 369:954-64.

11. Font-Mayolas, S., Gras, M. E. y Planes, M. (2006). Análisis del patrón de consumo de cannabis en estudiantes uni-versitarios. Adicciones, 18(4), 337-344.

12. García-Araiza HJ, Hernández-Chávez L, Och-Castillo JM, et al. (2019). Estrés universitario en estudiantes de medicina de la Universidad de Quintana Roo. Salud Quintana Roo. 2019;12(41):1317.

13. Hernández-Sampieri, R. \& Mendoza, C (2018). Metodología de la investigación. Las rutas cuantitativa, cualitativa y mixta, Ciudad de México, México: Editorial Mc Graw Hill Education, Año de edición: 2018.

14. Instituto Nacional de Salud Pública. (2013). Encuesta Nacional de Adicciones 2011: tabaco. México: INSP; 2013 http://www.conadic.salud.gob.mx/pdfs/investigacion/ENCODE_DR OGAS_2014.pdf 
15. Kenna, G. A., \& Lewis, D. C. (2008). Risk factors for alcohol and other drug use by healthcare professionals. Substance abuse treatment, prevention, and policy, 3, 3. https://doi.org/10.1186/1747-597X-3-3

16. Lara, N., Saldaña, Y., Fernández, N. y Delgadillo, H. J. (2015). Salud, calidad de vida y entorno universitario en estudiantes mexicanos de una universidad pública. Hacia la Promoción de la Salud, 20(2), 102117. http://www.scielo.org.co/pdf/hpsal/v20n2/v20n2a08.pdf

17. Leonor Alexandra Rodríguez Alava, Betty Alexandra Lino Moreira y Martha Cecilia Escobar (2017): “Estrés académico en psicólogos clínicos en formación”, Revista Atlante: Cuadernos de Educación y Desarrollo (noviembre 2017). http://www.eumed.net/rev/atlante/2017/11/estres-academico.html

18. Magaña, A. (2007). Relación del consumo de drogas ilícitas con el rendimiento académico (tesis de maestría, Uni-versidad Autónoma de Querétaro, México).

19. McAuliffe W, Rohman M, Breer P, Wyshak G, Santangelo S, Magnuson E. (1991). Alcohol use and abuse in random samples of physicians and medical students. American Journal of Public Health 1991; 81: 177-82.

20. Nieves, Z., Satchimo, A., González, Y. y Jiménez, M. (2013). Algunas consideraciones acerca del estrés académico en los estudiantes universitarios. Revista Psicoespacios, 7(11), 91-116. http://revistas.iue.edu.co/revistasiue/index.php/Psicoespacios/article/v iew/215

21. Obregón-Morales, Berea, Montalván-Romero, José C., SegamaFabian, Edinho, Dámaso-Mata, Bernardo, Panduro-Correa, Vicky, \& Arteaga-Livias, Kovy. (2020). Factors associated with depression in medical students from a peruvian university. Educación Médica Superior, 34(2), e1881. Epub 01 de junio de 2020. http://scielo.sld.cu/scielo.php?script=sci_arttext\&pid=S086421412020000200013\&lng=es\&tlng=en.

22. Organización Mundial de la Salud (2018). 10 amenazas a la salud mundial en 2018. https://www.who.int/features/2018/10-threatsglobal-heath/es/

23. Restrepo, J. E., Sánchez, O. A., \& Castañeda Quirama, T. (2020). Estrés académico en estudiantes universitarios. Psicoespacios, 14(24), 17-37. https://doi.org/10.25057/21452776.1331

24. Román Collazo, C. A., Ortiz Rodríguez, F., \& Hernández Rodríguez, Y. (2008). El estrés académico en estudiantes latinoamericanos de la carrera de Medicina. Revista Iberoamericana De Educación, 46(7), 18. https://doi.org/https://doi.org/10.35362/rie4671911 
25. Romero, M.I., Santander J., Hitschfeld, M.J., Labbé, M. \& Zamora, V. (2009). Consumo de sustancias ilícitas y psicotrópicos entre los estudiantes de medicina de la Pontificia Universidad Católica de Chile. Revista Médica de Chile. 137, 459-465. http://www.scielo.cl/scielo.php?script=sci_arttext\&pid=S003498872009000400002

26. Sánchez-Hoil A, Andueza-Pech MG, Santana-Carvajal AM, HoilSantos JJ, CuFarfán-López J. (2017). Características sociodemográficas y perfil de consumo de tabaco y drogas en estudiantes de dos universidades de México. Rev Biomed. 2017;1(28):11-27.

27. Santos Morocho, J.L., Jaramillo Oyervide, J.A., Morocho Malla, M.I., Senín Calderón, M.C. y Rodríguez Testal, J.F. (2017). Estudio trasversal: Evaluación del estrés académico en estudiantes de Medicina y su asociación con la depresión. Revista Médica HJCA, 9 (3), 255260.

28. Soto-Estrada, G, Moreno-Altamirano, L, \& Pahua Díaz, D. (2016). Panorama epidemiológico de México, principales causas de morbilidad y mortalidad. Revista de la Facultad de Medicina (México), 59(6), 8-22. Recuperado en 17 de junio de 2019, de http://www.scielo.org.mx/scielo.php?script=sci_arttext\&pid=S0026$17422016000600008 \& \operatorname{lng}=\mathrm{es} \&$ tlng=es.

29. Velásquez, C. y Mejía, J. (2009). Rendimiento académico y abusodependencia de sustancias psicoactivas en los estudiantes de la Universidad de Antioquia. Memorias ivclabes: Cuarta Conferencia Latinoamericana sobre el Abandono en la Educación Superior. https://goo.gl/cBvgou

30. Villatoro J, Medina-Mora ME, Fleiz C, Moreno M, Oliva N, Bustos M, et al. (2011). El consumo de drogas en México: Resultados de la Encuesta Nacional de Adicciones, 2011. Salud Mental 2012 Nov./Dic.; 35(6):447-457

31. Zárate-Depraect, Nikell E., Soto-Decuir, María G., Martínez-Aguirre, Eunice G., Castro-Castro, María L., García-Jau, Rosa A., \& LópezLeyva, Nidia M.. (2018). Hábitos de estudio y estrés en estudiantes del área de la salud. FEM: Revista de la Fundación Educación Médica, 21(3), 153-157. Recuperado en 03 de septiembre de 2019, de http://scielo.isciii.es/scielo.php?script=sci_arttext\&pid=S201498322018000300007\&lng=es\&tlng=es. 